\title{
Improving Pre-emptive Prescribing to Relieve Patient Discomfort Occurring Out of Hours
}

Rhys Williams, Fiona Herbert, Amy Orme, Georgina Casswell

University Hospitals Bristol NHS Trust, UK

\begin{abstract}
Junior doctors are commonly asked to prescribe simple medications for symptom relief for patients out of hours. Unfortunately, time constraints and other pressures may lead to delays before the medications are prescribed. A quality improvement project was conducted at a large university teaching hospital to establish the extent of the problem, with the aim of finding measures to improve preemptive prescribing for patients. Baseline data was gathered over three busy wards to calculate the total of new prescriptions made over the course of a weekend. There were 24 new prescriptions required over the weekend, a percentage increase of $14.9 \%$ compared to the existing prescriptions on a Friday. Following the first intervention this decreased to $10.2 \%$, and by the second intervention the rate was $4.9 \%$. Data collected several months later confirmed that the interventions remained successful, and preemptive prescribing continued. Overall, our interventions have shown that the number of new prescriptions required out of hours can be reduced by educating junior doctors on preemptive prescribing.
\end{abstract}

\section{Problem}

We are a group of foundation year one junior doctors who have recognised, over the course of our first year in medical practice, that much of our time out of hours is spent prescribing simple symptomatic relief for patients suffering from symptoms that could have been preempted. This contributes to a large proportion of bleeps to our pagers while on call, and can even delay the assessment of clinically unwell patients.

\section{Background}

It is well recognised that patient morbidity and mortality rates are higher at weekends and out of hours, a phenomenon popularly known as the "weekend effect."[1] This difference in outcomes has been documented for multiple conditions across many specialties, from respiratory medicine to trauma and orthopaedics. The weekend effect has been attributed primarily to limited staffing levels out of hours, meaning that deteriorating patients may not be identified and assessed as promptly.[1]

Bleeps to junior doctors on call are notoriously frequent. One study in New Zealand found that bleeps may be received as often as every seven minutes.[2] Many of these calls are related to basic medical activities including prescribing medications and intravenous fluids.[3] Answering and triaging these calls limits doctors' abilities to act on these tasks in a timely manner, and may even delay and interrupt assessment of unwell patients.[4] It is already known that likelihood of survival from an in hospital cardiac arrest is lower out of hours, and it has been postulated that this is due to reduced staffing levels, in addition to other factors.[5]

Several studies from emergency departments have shown that patients wait longer for analgesia when the department, and therefore members of staff, are busier.[6] Patients already wait longer than they would both like and expect for symptomatic treatments such as analgesia in the emergency department,[7] and this may also be the case among inpatients. As staffing levels are lower out of hours, and those staff present are often busier than at other times, this exacerbates the problem of delayed prescribing from both a patient safety and patient comfort perspective.

With this in mind, the aim of our quality improvement project was to reduce the impact of poor preemptive prescribing on both patients and staff members, by encouraging staff to consider anticipatory prescribing of symptomatic treatments prior to the weekend.

\section{Baseline measurement}

Before our first intervention, baseline data was collected from the drug charts of 56 patients across three wards encompassing medicine (A400), surgery (A800), and cardiology (C805) at the Bristol Royal Infirmary, Bristol, UK. The number of analgesics, laxatives, and antiemetics prescribed both prior to and following the weekend were counted. Both regular and "as required" (PRN) medications were included. All patients on these three wards were included unless they were admitted partway through the weekend, or if their drug charts were unavailable at the time of data collection. Our results demonstrate that there were 24 new prescriptions across the three wards, a percentage increase of $14.9 \%$ of the total presciptions for analgesics, laxatives, and antiemetics written on these wards.

\section{Design}

An informal discussion with foundation doctors at a mandatory trust teaching identified that many other junior doctors working in specialties across the hospital had experienced similar problems, but had no clear idea of how it could be rectified. We therefore felt that an educational intervention to raise awareness of the issue and 
encourage preemptive prescribing would be most effective.

\section{Strategy}

PDSA cycle 1

At a foundation doctor mandatory teaching session we gave a brief oral presentation on the problem, and encouraged doctors to consider incorporating patient-specific preemptive prescribing into their pre-weekend routines, alongside weekend handovers and pathology requests for example. We observed a substantial reduction in prescriptions for symptomatic treatments over the weekend on the medical wards. There was however minimal improvement on the surgical ward following our initial intervention.

PDSA cycle 2

In order to better target doctors working in surgery we next distributed an email to all doctors in surgical foundation posts, reminding them to consider anticipatory prescribing before the weekend, and notifying that medical wards are outperforming surgical wards in this respect.

\section{Results}

Following our first intervention the total percentage of new prescriptions required across the weekend over all three wards decreased from $14.9 \%$ to $10.2 \%$ (figure 1 ). The number of prescriptions decreased for all three types of medication: analgesia, laxatives, and antiemetics. This improvement was most pronounced on the cardiology ward, where no new prescriptions were written over the weekend (figure 2).

After our email to the surgical junior doctors there was a further reduction in the overall number of weekend prescriptions on the surgical ward, from $8.8 \%$ to $6.5 \%$, and across the three wards as a whole, from $10.2 \%$ to $4.9 \%$.

The figure graphs show the percentage (top panel) and absolute number (bottom panel) of new prescriptions of analgesics, antiemetics, and laxatives being written over the weekend, both overall and on each of the three individual wards. Percentages are derived from the proportion of total prescriptions for these medications that were newly made over the weekend.

See supplementary file: ds6951.pdf - "Percentage (top panel) and absolute number (bottom panel) of new weekend prescriptions, overall and on each ward."

\section{Lessons and limitations}

Our findings suggest that the number of additional symptomatic treatments needing to be prescribed by on call staff over the weekend can be substantially reduced by very simple educational measures. We theorise that this effect would prevent an unnecessary period of suffering for patients with ongoing discomfort while waiting for remedies to be prescribed. In addition we feel this decreased workload for on call staff would reduce fatigue, and provide more time to concentrate on more demanding tasks such as the management of unwell patients.[8] Despite political suggestions of a movement towards a truly "seven day NHS," at present this prospect seems unlikely to materialise before the distant future. Therefore, the "weekend effect" is likely to remain an ongoing problem for both staff and patients in the UK, at least in the medium term. We feel simple measures such as those in this study are an effective way to instigate improvement in outcomes relating to patient care over the weekend.

There are significant limitations to our methodology inherent to any quality improvement project. Our findings relate to a relatively small patient cohort on a few wards within a specific teaching hospital and at precise moments in time, and the cross sectional nature of the study limits its external validity. The decision to sample patients across contrasting wards in medicine and surgery was made with the intention to minimise this limitation, however.

More specific to our study design is the potential confounding influence of improvements in anticipatory prescribing that could be expected to be seen among junior doctors as they develop as clinicians with general experience, particularly during the first year of practice. The reduction in additional prescribing required over the weekend seen on a medical ward following our surgery specific second intervention may illustrate this phenomenon. Alternatively, however, it could be due to an element of sample cross contamination that occurs when junior doctors on both medicine and surgery discuss local active quality improvement projects together, and as they rotate across different specialties and wards throughout the year.

Furthermore, educational interventions have been associated with only transient improvements in outcomes.[9] With this is mind we collected further data two months following our second intervention, in order to assess the sustainability of the improvements we have observed, which showed continued improvement. We recognise that in order to make more permanent change, regular reeducation is necessary. We are therefore incorporating our educational measures into the compulsory trust induction courses undertaken annually by junior doctors as of August 2016, and in the trust induction booklet distributed on this occasion. Further educational measures to consider could target other clinicians whose influence could improve preemptive prescribing, including pharmacists and nursing staff.

Future projects with an alternative means of reinforcing our message in the longer term could include the incorporation of a prompt to consider preemptive prescribing on clerking proformas or weekend handover sheets. Pre-printed labels could be used to potentially save time in prescribing symptomatic relief on busy Friday ward rounds, but at significant financial cost. Projects may also consider the use of alternative outcome measures relating to staff workload and quality of patient care at the weekend, for instance the number of bleeps received by on call staff relating to requests for simple prescriptions, or the delay between the request for symptomatic relief and its administration once the prescription is written by on call staff. Furthermore, it might be useful to monitor 
the number of prescription errors made by the time pressured on call team lacking knowledge of patients' backgrounds, compared to the usual ward team. If found to be beneficial, interventions used to make improvements at the weekend could potentially be adapted and used to the benefit of out of hours services overnight.

\section{Conclusion}

A large proportion of junior doctors' workloads is recognised to consist of prescriptions of simple medications, which on some occasions could be better preempted. We found that education of junior doctors substantially reduced the number of prescriptions required at the weekend across both medical and surgical specialties at a large university teaching hospital, and that the effect continued several months following intervention.

\section{References}

1. Bell CM, Redelmeier DA. Mortality among patients admitted to hospitals on weekends as compared with weekdays. N Engl J Med 2001;345(9):663-8.

2. Chiu T, Old A, Naden G, Child S. Frequency of calls to "oncall" house officer pagers at Auckland City Hospital, New Zealand. N Z Med J 2006;119(1231):U1913.

3. Morton J, Williams Y, Philpott M. New Zealand's Christchurch Hospital at night: an audit of medical activity from 2230 to 0800 hours. N Z Med J 2006;119(1231):U1916.

4. Blum NJ, Lieu TA. Interrupted care. The effects of paging on pediatric resident activities. Am J Dis Child 1992;146(7):806-8.

5. Peberdy MA, Ornato JP, Larkin GL, et al. Survival from inhospital cardiac arrest during nights and weekends. JAMA 2008;299(7):785-92.

6. Mills AM, Edwards JM, Shofer FS, Holena DN, Abbuhl SB. Analgesia for older adults with abdominal or back pain in emergency department. West J Emerg Med 2011;12(1):43-50.

7. Fosnocht DE, Swanson ER, Barton ED. Changing attitudes about pain and pain control in emergency medicine. Emerg Med Clin North Am 2005;23(2):297-306.

8. Peate I. The weekend effect and compassion fatigue. $\mathrm{Br} \mathrm{J}$ Nurs 2014;23(20):1059.

9. Tabali M, Jeschke E, Bockelbrink A, et al. Educational intervention to improve physician reporting of adverse drug reactions (ADRs) in a primary care setting in complementary and alternative medicine. BMC Public Health 2009;9:274.

\section{Declaration of interests}

Nothing to declare.

\section{Acknowledgements}

Project group members: Dr Fiona Herbert, Dr Amy Orme, Dr Rhys Williams. Each project member made an equal contribution to the study design, collection of data and project report.

Supervisor: Dr Jim Portal, Consultant Hepatologist.

Mentor: Dr Georgina Casswell, Registrar in Oncology.

The persons named above, plus the ward charge nurse and teams for agreeing to participate.

\section{Ethical approval}

According to the policy activities that constitute research at University Hospitals Bristol NHS Foundation Trust, this work met criteria for quality improvement rather than clinical audit or research, and was therefore not subject to ethical review though either of those frameworks. 